\title{
ERRATUM
}

\section{Mannose-Binding Lectin Is a Disease Modifier in Clinical Malaria and May Function as Opsonin for Plasmodium falciparum- Infected Erythrocytes}

Peter Garred, Morten A. Nielsen, Jørgen A. L. Kurtzhals, Rajneesh Malhotra, Hans O. Madsen, Bamenla Q. Goka, Bartholomew D. Akanmori, Robert B. Sim, and Lars Hviid

Tissue Typing Laboratory-7631, Department of Clinical Immunology, and Centre for Medical Parasitology, Department of Infectious Diseases, and Department of Clinical Microbiology, Rigshospitalet, Copenhagen, Denmark; RA Biology

Respiratory and Inflammation CEDD, GlaxoSmithKline Medicines Centre Gunnels Wood, Stevenage, and Medical Research Council Immunochemistry Unit, Department of Biochemistry, Oxford University, Oxford, England; and Department of Child Health, Korle-bu Teaching Hospital, Accra, and Immunology Unit, Noguchi Memorial Institute for Medical Research, Legon, Ghana

Volume 71, no. 9, p. 5245-5253, 2003. Page 5248, Table 3, column 6, row 3: "15,765" should read "115,765." 